\title{
Early Surgical Treatment of Pectoralis Major Tendon Rupture: A Case Report
}

\author{
Lim CT, MRCS (Ed), Wee J*, MRCS (Ed), Lee KT*, FRCS (Orth) (Ed) \\ University Orthopaedic Hand and Reconstructive Microsurgery Cluster, National University Health System, \\ Singapore \\ *Department of Orthopaedic Surgery, Tan Tock Seng Hospital, Singapore
}

\begin{abstract}
Pectoralis major rupture is a relatively uncommon injury. It is often under- or misdiagnosed due to late presentation and the lack of obvious clinical signs. Timely diagnosis and treatment is crucial, as early surgical repair has been shown to improve functional and cosmetic outcomes. We report the case of a young patient who underwent surgical repair of a right pectoralis major rupture sustained during a fall while wakeboarding. At the one-year follow-up, he had achieved full range of right shoulder motion and returned to his previous level of sporting activities.
\end{abstract}

Key Words:

Pectoralis major, anterior axillary fold, suture anchor

\section{INTRODUCTION}

Pectoralis major rupture is a relatively uncommon injury. The first case in the literature was reported by Patissier et al. in 1822. Pectoralis major ruptures have been described in work-associated accidents and horseback riding related events. More recently, pectoralis major ruptures resulting from indirect injuries during sports activities (i.e., bench presses) have been described ${ }^{1}$. This relatively uncommon injury is under-diagnosed due to its rarity, limited clinical signs and delayed presentation, especially when it occurs among patients involved in low workload activities. We present a rare case of pectoralis major rupture sustained during a wakeboarding accident.

\section{CASE REPORT}

A 32-year-old Chinese man who was previously well, presented with right shoulder pain after a fall into the water while wakeboarding eight days earlier. During the incident, he fell sharply into the water resulting in forced abduction and external rotation of his right shoulder. He thought he felt a 'snap' in his right shoulder during the fall. Bruises over the proximal anteromedial aspect of his right arm developed two days after the incident.
On examination, there was ecchymosis over the anteromedial aspect of the right arm and tenderness on palpation at the bicipital groove region. There was partial loss of the right anterior axillary fold which was accentuated by right shoulder abduction (Figure 1). Right shoulder movements were limited by pain. Magnetic resonance imaging (MRI) showed rupture of both the sternal and clavicular attachments of pectoralis major muscle with retraction of at least $5 \mathrm{~cm}$.

In view of his young age and high functional demand, we decided to perform an open repair of the pectoralis major rupture. Surgery was performed via a deltopectoral approach in a beach-chair position. The clavicular head was found to be ninety per cent ruptured near the insertion while the sternal head was completely ruptured near the musculotendinous junction. A burr was used to roughen the lateral lip of the bicipital groove; three $5.5 \mathrm{~mm}$ biocorkscrew suture anchors (Arthrex) were then inserted (Figure 2). The distal ends of the clavicular and sternal heads were secured with No. 2 fibre wires from the bio-corkscrews using Krackow stitches and anchored firmly onto the lateral lip of the bicipital groove.

His right upper limb was placed in an arm sling for 6 weeks postoperatively to protect the repair as well as for pain relief. Right shoulder passive range of motion exercise was started two weeks postoperatively, progressing to active movement at six weeks. Postoperatively, the anterior axillary fold was recreated (Figure 3). At a one-year follow-up, the patient had achieved full range of right shoulder motion and returned to his sporting activities without problems. The Constant Shoulder Score was 98 out of 100 .

\section{DISCUSSION}

There have been only about 200 cases of pectoralis major rupture reported to date. This injury is frequently under- or misdiagnosed because of its rarity, relatively few clinical signs, presence of only an isolated sternal head tear and minimal functional disability. 


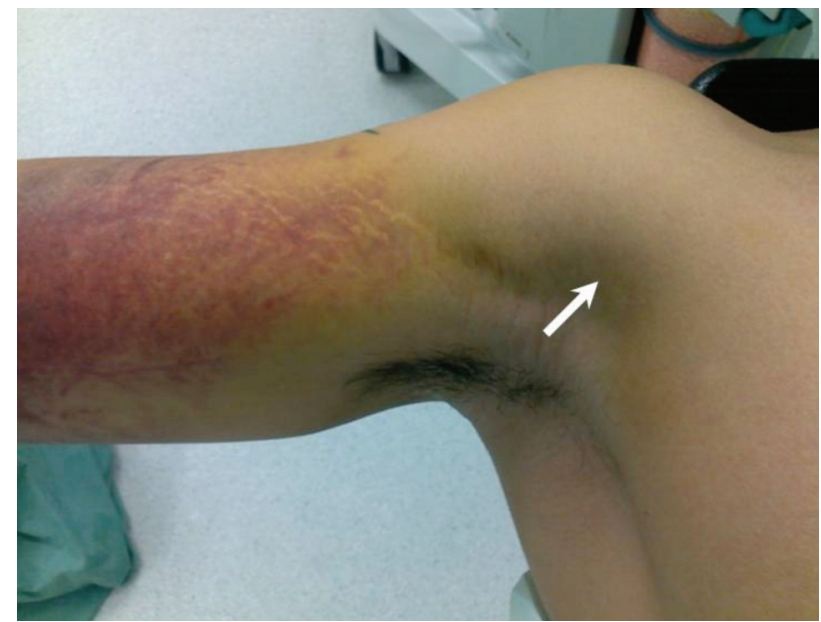

Fig. 1: Loss of anterior axillary fold in our patient with pectoralis major rupture.

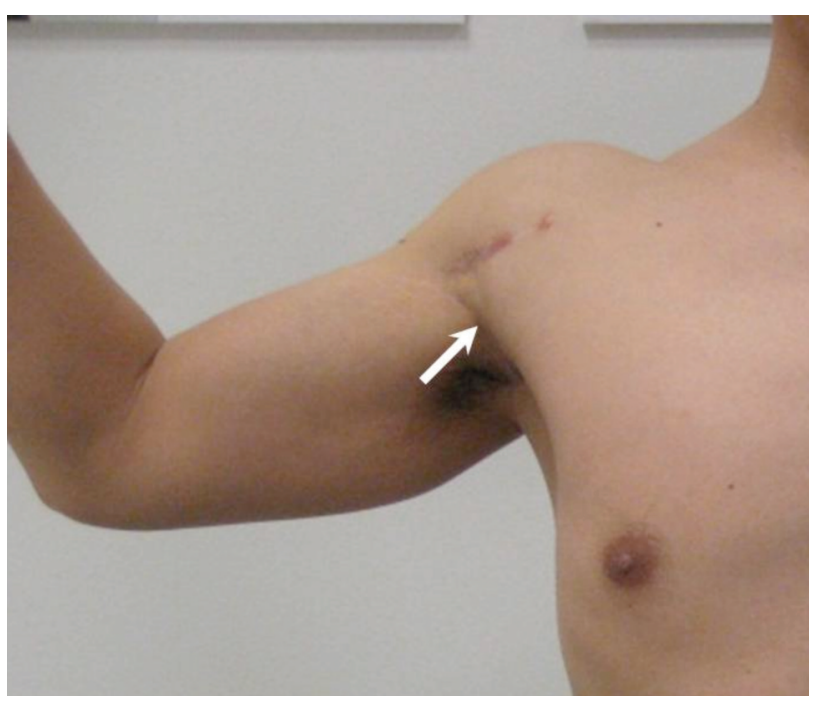

Fig. 3: Restoration of anterior axillary fold after surgery.

As an isolated injury, pectoralis major rupture results in limited functional reduction during activities of daily living. Patients normally present with ecchymosis and/or tenderness around the affected region with pain on active adduction and internal rotation of the injured shoulder. A more specific sign for pectoralis major rupture is the loss or thinning of the anterior axillary fold. The defect can be accentuated by abduction ${ }^{2}$, but also may be masked by associated swelling and haematoma. With isolated sternal or clavicular head rupture, the remaining intact head would preserve the anterior axillary fold.

Wakeboarding is a form of surface water sport in which a participant rides on a floating board towed at high speed by a motorboat. It is considered safe even for beginners because the participant wears a lifejacket, and is therefore unlikely to get seriously injured when he loses balance. However, as illustrated in our case, falling into the water at high speed can result in significant impact to an abducted shoulder.

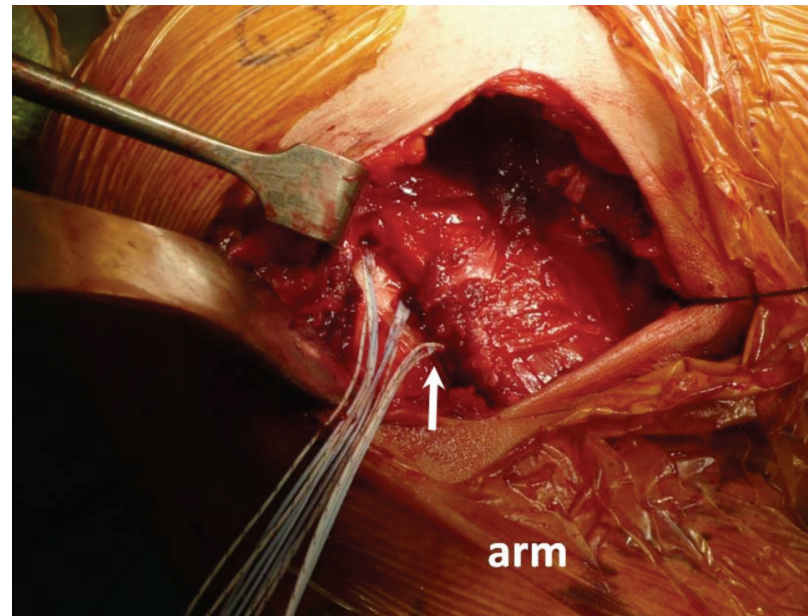

Fig. 2: Three bio-corkscrews suture anchors (arrow) inserted along lateral lip of bicipital groove in preparation for repair of the pectoralis major muscle.

It is important to have patients undress and expose the shoulder for examination. Unless done so, loss of anterior axillary fold could be easily missed. Routine strength testing of only the rotator cuff and deltoid muscles will not detect any loss in power as well, especially when shoulder pain is reduced due to late presentation. Without a high index of suspicion, bruising may be mistaken for a simple contusion. This case thus illustrates the importance of a complete physical examination, including looking, palpating and assessing range of motion, for any shoulder injury.

The two most common techniques for repair of pectoralis major rupture are the use of transosseous sutures and suture anchors. Although the transosseous technique has long term evidence of good results ${ }^{3}$, it is associated with tendon shortening (average of $9 \mathrm{~mm})^{4}$. Using suture anchors allows for less extensive soft tissue stripping and reduced operative time. In addition, any native tendon on the humeral side can be preserved and used to reinforce the repair ${ }^{4}$, yielding similar stiffness to transosseous repair. With its proven mechanical strength, we utilized this surgical technique in the present case.

Early diagnosis of pectoralis major rupture is crucial so that early surgical treatment can be performed. In a meta-analysis by Bak et $a l^{5}, 88 \%$ of the patients treated surgically had excellent/good results while only $27 \%$ of those treated conservatively achieved excellent/good results. That study also showed that $57 \%$ of those surgically treated within eight weeks of injury obtained excellent results compared to $16 \%$ who had delayed surgical repair. Surgically treated patients also returned to $99 \%$ peak torque of the uninjured upper limb while those treated conservatively only achieved $56 \%$ of the uninjured side. We chose surgical repair for our young patient to restore his strength and achieve maximum functional outcome. 
In summary, pectoralis major rupture is a relatively uncommon injury. Diagnosis can often be missed due to late presentation and lack of obvious clinical signs. Timely diagnosis with a high index of suspicion is crucial, as early surgical treatment yields the best functional and cosmetic results.

\section{REFERENCES}

1. Provencher MT, Handfield K, Boniquit NT, Reiff SN, Sekiya JK, Romeo AA. Injuries to the pectoralis major muscle: diagnosis and management. Am J Sports Med 2010; 38(8): 1693-705.

2. Hayes WM. Rupture of the pectoralis major muscle; review of the literature and report of two cases. J Int Coll Surg 1950; 14(1): 82-8.

3. Schepsis AA, Grafe MW, Jones HP, Lemos MJ. Rupture of the pectoralis major muscle. Outcome after repair of acute and chronic injuries. Am J Sports Med 2000; 28(1): 9-15.

4. Hart ND, Lindsey DP, McAdams TR. Pectoralis major tendon rupture: a biomechanical analysis of repair techniques. J Orthop Res 2011; 29(11): 1783-7.

5. Bak K, Cameron EA, Henderson IJ. Rupture of the pectoralis major: a meta-analysis of 112 cases. Knee Surg Sport Tr A 2000; $8(2): 113-9$ 